\title{
Alternate update of shifted extended keyholes (AUSEK): A new accelerating strategy for interventional MRI
}

Shuai Yu ${ }^{\text {a }}$, Derek Kwok ${ }^{\text {b }}$, Yufu Zhou ${ }^{\text {a }}$, Qing Zhang ${ }^{a}$, Xiaoyan Huang ${ }^{\text {a }}$, Geng Li ${ }^{\text {b }}$, Bensheng Qiu ${ }^{\text {a,* }}$

${ }^{a}$ Center for Biomedical Engineering, University of Science and Technology of China, Hefei, Anhui 230027, China

b Time Medical Limited, Shatin N.T., Hong Kong 999077, China

* Corresponding author. Department of Electronics Science and Technology, University of Science and Technology of China, P.O. Box 4, Hefei, Anhui 230027, China;

E-mail address: bqiu@ustc.edu.cn (B. Qiu) 


\begin{abstract}
Real-time or near-real-time acquisition plays a key role in providing immediate image guidance for interventional magnetic resonance imaging (iMRI). However, the requirement of accurate needle tip localization has made several accelerating techniques, like Keyhole imaging or sliding window reconstruction, difficult to be applied to iMRI. The purpose of this work was to further explore the possible ways of applying view sharing techniques to iMRI. Inspired by Keyhole imaging, we present an easy-toimplement accelerating strategy called "Alternate update of shifted extended keyholes (AUSEK)". In this method, the keyhole views are not only extended but also shifted towards either high-frequency edge to form two divisions in k-space. The divisions which are mirrored to each other along the center are alternately updated following a reference scan. By using simulations and experiments, we demonstrate that AUSEK could effectively preserve the spatial resolution of the image, especially of the needle, at a temporal acceleration rate of about 2.5. AUSEK was implemented online in an openconfiguration low-field MR imaging system.
\end{abstract}

Keywords: Interventional MRI; Accelerated imaging; Keyhole; k-space substitution 


\section{Introduction}

Over the years, the demand for MR-guided minimally invasive surgeries keeps growing and the techniques of interventional MRI (iMRI) have advanced rapidly. High tissue contrast, no risk of ionizing radiation, multiple contrast mechanisms, arbitrary plane orientation and temperature sensitivity seem to have made MRI the perfect modality for image guidance in interventional procedures. IMRI has been used in procedures like spinal injection [1-3], joint puncture and arthrography [4-6], liver tumor ablation [7-9], breast cancer therapy [10,11] and so on. Although many improvements have been made on the gradient system, the acquisition speed of MRI is still slower compared with other imaging modalities such as computed tomography (CT) or ultrasound. This is especially true for low-field scanners.

The spatial redundancy in k-space could be exploited to design an efficient sampling scheme. And for many interventional procedures, especially when the interventional device is pretty close to the target, freeze mode, that is images are continuously updated with a fixed FOV, is used [4]. In cases like this, the temporal redundancy in k-space could be exploited, too. View sharing methods, like Keyhole imaging $[12,13]$ or sliding window reconstruction, are used to improve temporal resolution of MRI for many years [14-16]. But their applications to iMRI seem not very straightforward. Interleaved multiturn radial sampling was used with sliding window reconstruction for real-time MRI in [16]. But for iMRI, sliding window reconstruction with multiple intermediate images may be inherently not suitable for iMRI. Take the needle insertion as an example. During the interventional procedure, the needle is almost always moving, usually at a speed of 2 $\mathrm{mm} / \mathrm{second}$. So for sliding window reconstruction, the reconstructed needle image of current update would overlay the one of previous updates. But only the current update contains relevant information of the true current needle tip position. There would be several intermediate darkness levels at the tip part of the needle if multi-turn sliding window reconstruction was used and the darkness level of the tip would be the lowest among them. If too many turns were used, the tip might be too obscure to be noticed. The application of Keyhole imaging to iMRI was studied by Jeffrey L. Duerk in [14, 17]. When the phase encoding direction is perpendicular to the needle shaft, the spatial 
resolution, especially the width resolution of the needle shaft would be inadequate for conventional keyhole method which repeatedly updates the center $25 \%$ of k-space. When the phase encoding direction is parallel to the needle shaft, although the width resolution is excellent, the ringing artifacts at the tip would make the conventional keyhole method difficult to be applied to iMRI. From another perspective, the contradiction of using conventional Keyhole imaging is that only updating the low-frequency part would lose too much critical high-frequency information about the needle. Hence, we were inspired by [14-17] and proposed a simple updating scheme called "alternate update of shifted extended keyholes (AUSEK)".

The purpose of this study was to further explore the possible ways of applying view sharing techniques to iMRI while preserving adequate spatial resolution of the image, especially of the needle. Simulation experiments, dynamic phantom experiments and ex vivo experiments were conducted. AUSEK was implemented online in an openconfiguration, low-field MR imaging system.

\section{Materials and methods}

\subsection{Materials}

A phantom was used in the experiments. The materials used for this phantom were Agarose (4\%), $\mathrm{NaCl}(0.5 \%), \mathrm{CuSO} 4-5 \mathrm{H} 2 \mathrm{O}(0.05 \%)$ and distilled water. The phantom was roughly in the shape of a cuboid. The length, breadth and height of the phantom were about $16 \mathrm{~cm}, 12 \mathrm{~cm}$ and $8.5 \mathrm{~cm}$ respectively. Two tubes were placed in the phantom. The diameter of the thin one with air filled in it was about $0.5 \mathrm{~cm}$. A $16-\mathrm{G}$ MR-compatible needle with a $20^{\circ}$ bevel tip was used. It was constructed of a Nickel-Titanium alloy. One pig thigh was used for needle insertion in ex vivo experiments.

All imaging was done on a $0.35 \mathrm{~T}$ open-configuration whole-body MR scanner (PICA, Time Medical Systems, Hong Kong, China). A receive-only quadrature knee coil was used. This coil was custom-made for intervention. The interventional MRI system consisted of the MR scanner, two in-room monitors, one infrared camera, fixed infrared reflector reference to enable frameless stereotaxy. Optical tracking was performed using the infrared navigator camera (NDI, Waterloo, Ontario, Canada), like in [18]. The 
simulation experiments were done using MATLAB (The Mathworks, Natick, MA). The dynamic phantom experiment required at least two people working in coordination in the scanning room. One person was performing the procedure and the other one was controlling the pulse sequences.

\subsection{The process of simulation experiments}

Inspired by the simulation study done by Jeffrey L. Duerk in [17], we designed a similar one (Fig. 1). The simulation experiments started with an axial image of the phantom. This image was reconstructed from a fully-sampled raw data which was acquired by a conventional spin echo pulse sequence (1 slice, TR $450 \mathrm{msec}$, TE $15 \mathrm{msec}$, slice thickness/interval $4 \mathrm{~mm} / 2 \mathrm{~mm}$, field of view (FOV) $256 \mathrm{~mm} \times 256 \mathrm{~mm}$, matrix 256 $\times 256$, ETL 1, acquisition time 1 minutes, 55 seconds) on the 0.35T scanner. As in [17], we got the original raw data by the inverse two-dimensional Fourier transform (2DFT) of the SE magnitude image. Then a two-pixel-wide simulated needle, consisting of zerovalue pixels, was "inserted" into the phantom image. At the needle tip (the right end), the lower row of the needle had one more pixel than the upper row to simulate a $45^{\circ}$ bevel tip. The simulated needle's insertion direction was set as both directly towards the target of the dark dot (the air-filled thin tube) and perpendicular to the phase encoding direction [17]. We got the raw data of this image by 2DFT and sampled a selected section of it and replaced the corresponding part of the original raw data. For different set of comparison, the specific sampling pattern may be different. After 2DFT of the updated raw data, we got the updated image with a needle in it. This concludes the first cycle. Then we replaced the original image with the updated image and advanced the simulated needle at the same time. The needle insertion rate was set as 6 pixels per cycle. An insertion cine was generated as the cycle was repeated 4 times. In addition to the cycle, in the updated image reconstructed following the k-space update, we also attached another simulated needle (same width, same length, same shape and same direction) parallel to the "insertion" location for comparison. The images with reference needles in them were only used for evaluation and were not included in the repetition cycle.

\subsection{Simulation experiments}

\subsubsection{Comparison between four different updating schemes}


The four updating schemes (Fig. 2) were inspired by Keyhole imaging [12-14, 17] and sliding window reconstruction. The first scheme (Fig. 2a) only updated one single division in k-space repeatedly (we called it "repeatedly single-division updating scheme"). This division contained $40 \%$ of the full number of views. There were two divisions which got updated alternately in the second scheme (AUSEK) (Fig. 2b). Either division contained $40 \%$ of the full number of views and was mirrored to the other along the center. In the third scheme (Fig. 2c), interleaved two-turn updating was used. The total views which got updated in two turns constituted $80 \%$ of the full number of views. In the fourth scheme (Fig. 2d), interleaved three-turn updating was used. The total views which got updated in three turns constituted $80 \%$ of the full number of views.

\subsubsection{Comparison between different levels of shifting}

In this set of experiments, we compared the images when different levels of shifting were set in AUSEK. The percentage of either of the updating divisions was fixed as $40 \%$. We denoted the shifting level by the smaller updating percentage in either half of k-space / the larger updating percentage in either half of k-space. Four levels of shifting, which were $20 \% / 20 \%, 10 \% / 30 \%, 5 \% / 35 \%$ and $0 \% / 40 \%$, were used for comparison. The corresponding root-mean-square (RMS) differences of the whole image with needle pixels included, of the whole image with needle pixels excluded, of the needle part with needle pixels included and of the needle part with needle pixels excluded between the reconstructed images and the original one were calculated. The needle part was defined as a part of the image which surrounded the needle pixels. In the direction of the needle's long axis, every row of this part was two pixels longer respectively (one pixel on each side). Every column of this part was also six pixels wider than the attached needle respectively (three pixels on each side). The needle pixels were the corresponding pixels of the precise needle "insertion" location.

\subsubsection{Comparison between different levels of division size}

In this set of experiments, we compared the images when different number of views in the division were chosen using AUSEK. Four levels of division size, which were 20\%, $30 \%, 40 \%$ and $50 \%$, were chosen. The shifting level was fixed as the smaller updating percentage in either half of $\mathrm{k}$-space was $0 \%$. The corresponding RMS differences of the 
whole image with needle pixels included, of the whole image with needle pixels excluded, of the needle part with needle pixels included and of the needle part with needle pixels excluded between the reconstructed images and the original one were calculated.

\subsection{Dynamic phantom experiments}

AUSEK implemented with FLASH (fast low-angle shot) pulse sequence was used to accelerate acquisition after the needle was inserted a little bit. We set the division size as $40 \%$ and the shifting level as $10 \% / 30 \%$. Freeze mode, under which images were updated continuously with a fixed FOV, was kept on during the following insertion. Note that the phase encoding direction was perpendicular to the needle insertion direction. A fully-sampled reference scan was done first. Then the needle insertion was performed in the direction towards the assumed target of the dark dot (the air-filled thin tube) at an insertion rate of about $5 \mathrm{~mm}$ per scan. The acquisition parameters were as follows: 3 slices, TR/TE $21.33 / 8.76 \mathrm{msec}$, flip angle $36^{\circ}$, filed of view (FOV) $256 \mathrm{~mm} \times 256 \mathrm{~mm}$, receiver bandwidth $98 \mathrm{~Hz} /$ pixel, matrix size $256 \times 256$, slice thickness $5 \mathrm{~mm}$. Images of three slices were updated at once every 6.6 seconds. During the needle insertion, the angle between the needle insertion direction and the main field direction was about $40^{\circ}$. Note that the phantom was placed askew in the coil.

\subsection{Ex vivo experiments}

Almost the same process as in the dynamic phantom experiments was used for needle insertion in an ex vivo pig thigh. Only the target was chosen as the small bone (kneecap, also called patella) in the image before insertion.

\section{Results}

\subsection{Simulation experiments}

\subsubsection{Comparison between four different updating schemes}

Figure 3 shows that the shapes of needle are pretty well preserved in all four updating schemes. For scheme 1 (repeatedly single-division updating scheme), the needle's darkness is very consistent but is also much less than the attached reference needle. It can also be corroborated by the RMS differences of different parts (Table 1). When needle 
pixels are included, the RMS differences of scheme 1 are the largest of all four. But when needle pixels are excluded, the RMS differences of scheme 1 become the smallest of all four. For scheme 2 (AUSEK), both the image and the needle's shape are very similar to the first scheme. Only the needle's darkness is not consistent. At the tip, the darkness is much less than the part closer to the needle butt. It can be seen that there is a one-stairstep decline of the needle's darkness. When needle pixels are included, the RMS differences (Table 1) of scheme 2 are the least of all four. When needle pixels are excluded, the RMS differences (Table 1) of scheme 2 are very close to the least of all four (scheme 1). Hence, we think AUSEK is the best of all four considering the spatial resolution of the image, especially of the needle. For scheme 3 (interleaved two-turn updating scheme), there is also a one-stairstep decline in the needle's darkness. Besides, there is one extra parallel needle "flying" somewhere above. In this extra needle, you can see there are two changes from darkness to lightness. It corroborates with the fact that this is the image of the fourth update. Because of the artifacts above, the RMS differences (Table 1) of this scheme is larger than AUSEK. For scheme 4 (interleaved three-turn updating scheme), there is a two-stairstep decline of the needle's darkness. Besides, there are two extra parallel needles, which have gradations of darkness in them, "flying" somewhere above or below. Because of the artifacts above, the RMS differences (Table 1) of this scheme is larger than AUSEK.

\subsubsection{Comparison between different levels of shifting}

Figure 4 shows that as the division is more shifted towards either high-frequency edge, the ringing artifacts and the blurring artifacts of the needle become less severe but the needle's darkness also becomes less. When the shifting level is 0\% / 40\%, these artifacts are almost gone and we can spot the needle's shape very easily. These observations can be corroborated by the RMS differences of different parts (Table 2). Since the images as whole are pretty much the same, the RMS differences are most influenced by the needle and its artifacts. When the needle pixels are included, we can see that the RMS differences first decrease then increase as the shifting level gets higher. The mitigation of ringing artifacts and blurring artifacts would reduce the RMS differences. However, the decline of the needle pixels' darkness would increase the RMS differences. When the 
shifting level is low, still a large proportion of center views are sampled and the mitigation of ringing artifacts and blurring artifacts overcomes the decline of needle pixels' darkness. But when the shifting level gets higher, so many center views are missed that the decline of needle pixels' darkness makes the RMS differences become larger. But visually, the needle's shape is preserved almost the same as the original shape. When the needle pixels are excluded, we also exclude the influencing factor of darkness decline. The alleviation of ringing artifacts and blurring artifacts is solely studied. We can see that the ringing artifacts and the blurring artifacts are more mitigated and the needle's shapes are better preserved as the shifting level gets higher. The corresponding monotonic decrease of RMS differences (Table 2) also proves this.

\subsubsection{Comparison between different levels of division Size}

Figure 5 shows that as the division size gets bigger at the same shifting level, the blurring and the ringing artifacts of the needle become less severe. When the division size is $40 \%$, these artifacts are almost gone and we can observe the needle's shape very easily. These observations can also be corroborated by the RMS differences of different parts (Table 3). The RMS differences are almost always declining as the division size becomes bigger.

\subsection{Dynamic phantom experiments}

We set the shifting level as 10\% / 30\% instead of $0 \%$ / $40 \%$ because the needles in Figure 6 are much thicker than the ones in the simulation experiments. Two images updated (Fig. 6b and 6c) using AUSEK with FLASH immediately following needle advances show that there are ringing artifacts in the images and some blurring artifacts around the needle. But the $20^{\circ}$ bevel tips are preserved pretty well and can be easily spotted. Visually, no inconsistency of darkness at the tips can be seen.

\subsection{Ex vivo experiments}

There are also ringing artifacts and blurring artifacts in the images updated (Fig. 7b and 7c) immediately following needle advances. But the needle's shapes are still well preserved and we can spot the $20^{\circ}$ bevel tips easily. 


\section{Discussion and conclusions}

As demonstrated by the comparison among four different updating schemes, AUSEK can achieve the best spatial resolution of the image, especially of the needle. It can also be seen that inconsistency of needle's darkness would exist if multiple intermediate images were reconstructed with the sliding window reconstruction. Note that since we used only one image as the original image in the simulation experiments, the RMS differences are mainly determined by the needle's artifacts. For scheme 1 (repeatedly single-division updating scheme), although it is consistent, the needle's darkness is much less and a large proportion of center views are missed since it updates the same division in the same half of k-space repeatedly. We believe this would cause jerky or discontinuous movement in the image when the next fully-sampled reference scan is done. As demonstrated by the images (Figs. 4 and 5) and RMS differences (Tables 2 and 3), further shifting or more extension of the view division makes the ringing artifacts and the blurring artifacts of the needle more mitigated. Here we did not explore the cases of shifting the division past the center of k-space, since that would cause the most important center views not being updated.

We believe that setting the division size as about $40 \%$ of the full number of views and the smaller updating percentage in either half of $\mathrm{k}$-space as between $0 \%$ and $10 \%$ is a reasonable choice for interventional procedures using needles. The best choice can be determined based on the particular interventional environment, that is the shape, the size and compatibility of the interventional device, the main field strength, the particular pulse sequence this method is used with, the image quality physicians deem acceptable, even the orientation of the interventional device relative to the main field direction. In the dynamic phantom experiments, we inserted a 16-G MR-compatible needle using AUSEK with FLASH on a $0.35 \mathrm{~T}$ scanner. The needle insertion was done at about $40^{\circ}$ with the main field direction. The needles shown in the images were much thicker than the ones in the simulation experiments. So we set the shifting level as $10 \%$ / 30\% to include more center views instead of using $0 \% / 40 \%$ as in the simulation experiments. The darkness of the needle was pretty consistent and the needle tip could be spotted easily in both the dynamic phantom experiments and ex vivo experiments. 
The needle shaft could be rotated in a small degree during needle advance. The phase encoding gradient's shape may have varied a little bit between consecutive scans. The meat of pig thigh was not firm so its shape could be distorted by force to some extent during insertion. All these factors contributed some blurring artifacts and ringing artifacts in the updated images of dynamic phantom experiments and ex vivo experiments. The shifting level of $10 \% / 30 \%$ should have caused a small amount of ringing artifacts as demonstrated in the simulation experiments. Since the bulk of views ( $80 \%$ of the full number of views) were updated in every two consecutive updates, the blurring artifacts and ringing artifacts could be effectively eliminated by keeping the needle still for another update.

The biggest difference of designing an efficient sampling scheme between common MRI and interventional MRI is that you have to achieve an adequate spatial resolution of the moving needle for iMRI. And this difference has made several temporal acceleration techniques difficult to be applied to iMRI. For sliding window reconstruction, when multiple intermediate images are reconstructed, since only current update contains relevant information about the moving needle tip's true current position, the tip's darkness would be less and the tip's accuracy might be poor. In the study [17] done by Jeffrey L. Duerk, the conventional keyhole method which repeatedly updates the center $25 \%$ was proved not providing adequate spatial resolution. From [14, 17], it also can be inferred that acquisitions that acquire both high-frequency and low-frequency k-space data can provide acceptable tip position and needle width accuracy in both temporal and spatial domains for use in iMRI. Inspired by these findings, AUSEK was proposed. Not only sliding window reconstruction was used, but also both low-frequency and highfrequency k-space data were updated in AUSEK. We think adequate spatial resolution can be achieved by AUSEK, especially when FOV is fixed during the procedure. But technically, AUSEK does not count as a method of keyhole imaging, since the views in either division are much more.

One advantage of AUSEK is that it is very simple and easy-to-implement. In this method, Cartesian sampling trajectory is used. So only the phase encoding table of pulse 
sequence needs to be changed and before every reconstruction, the selected lines or columns in the matrix of raw data need to be replaced.

There are two limitations of this method. One is the inconsistency of needle tip's darkness as in the simulation experiments. But we update two divisions alternately and the shifting level is adjustable. We think the darkness sacrificed would not be much and the needle tip could still be spotted easily. In the dynamic phantom experiments and ex vivo experiments at a shifting level of $10 \% / 30 \%$, visually there was no inconsistency in the images. The other limitation is that the acceleration rate cannot be not very large. For the recommended division size of $40 \%$, the acceleration rate is only 2.5 . On the other hand, the cycle of reference scan can be much longer than in a typical keyhole method, since the bulk of views are updated much more frequently. Obviously, this scheme is more suitable for procedures during which FOVs can be fixed when a target enters.

In conclusion, alternate update of shifted extended keyholes (AUSEK) appears to be able to effectively preserve the spatial resolution of the image, especially of needle, at a temporal acceleration rate of about 2.5 . 


\section{Acknowledgements}

This work was supported by National Science Foundation of China (Grant number:

81371537, 91432301), Science and Technology Tackling Plan of Anhui and Major State Basic Research Development Program of China (973 Program) (Grant number:

2013CB733803). 


\section{References}

[1] Fritz J, Clasen S, Boss A, et al. Real-time MR fluoroscopy-navigated lumbar facet joint injections: feasibility and technical properties. European Radiology 2008; 18:1513-8. [2] Streitparth F, Walter T, Wonneberger U, et al. Image-guided spinal injection procedures in open high-field MRI with vertical field orientation: feasibility and technical features. European Radiology 2010; 20:395-403.

[3] Fritz J, Thomas C, Clasen S, Claussen CD, Lewin JS, Pereira PL. Freehand RealTime MRI-Guided Lumbar Spinal Injection Procedures at 1.5 T: Feasibility, Accuracy, and Safety. American Journal of Roentgenology 2009; 192:W161-W7.

[4] Hilfiker PR, Weishaupt D, Schmid M, Dubno B, Hodler J, Debatin JF. Real-time MRguided joint puncture and arthrography: preliminary results. European Radiology 1999; 9:201-4.

[5] Pereira PL, Gunaydin I, Trubenbach J, et al. Interventional MR imaging for injection of sacroiliac joints in patients with sacroiliitis. American Journal of Roentgenology 2000; 175:265-6.

[6] Soh E, Bearcroft PWP, Graves MJ, Black R, Lomas DJ. MR-guided direct arthrography of the glenohumeral joint. Clinical Radiology 2008; 63:1336-41. [7] Ricke J, Thormann M, Ludewig M, et al. MR-guided liver tumor ablation employing open high-field 1.0T MRI for image-guided brachytherapy. European Radiology 2010; 20:1985-93.

[8] Clasen S, Boss A, Schmidt D, et al. MR-guided radiofrequency ablation in a 0.2-T open MR system: Technical success and technique effectiveness in 100 liver tumors. Journal of Magnetic Resonance Imaging 2007; 26:1043-52.

[9] Clasen S, Pereira PL. Magnetic resonance guidance for radiofrequency ablation of liver tumors. Journal of Magnetic Resonance Imaging 2008; 27:421-33.

[10] Hynynen K, Pomeroy O, Smith DN, et al. MR Imaging-guided Focused Ultrasound Surgery of Fibroadenomas in the Breast: A Feasibility Study. Radiology 2001; 219:17685. 
[11] Gianfelice D, Khiat A, Boulanger Y, Amara M, Belblidia A. Feasibility of Magnetic Resonance Imaging-guided Focused Ultrasound Surgery as an Adjunct to Tamoxifen Therapy in High-risk Surgical Patients with Breast Carcinoma. Journal of Vascular and Interventional Radiology 2003; 14:1275-82.

[12] Jones RA, Haraldseth O, Müller TB, Rinck PA, Øksendal AN. K-space substitution: A novel dynamic imaging technique. Magnetic Resonance in Medicine 1993; 29:830-4. [13] Van Vaals JJ, Brummer ME, Thomas Dixon W, et al. "Keyhole" method for accelerating imaging of contrast agent uptake. Journal of Magnetic Resonance Imaging 1993; 3:671-5.

[14] Shankaranarayanan A, Wendt M, Aschoff AJ, Lewin JS, Duerk JL. Radial keyhole sequences for low field projection reconstruction interventional MRI. Journal of Magnetic Resonance Imaging 2001; 13:142-51.

[15] Uecker M, Zhang S, Voit D, Karaus A, Merboldt KD, Frahm J. Real-time MRI at a resolution of 20 ms. NMR Biomed 2010; 23:986-94.

[16] Zhang S, Block KT, Frahm J. Magnetic Resonance Imaging in Real Time: Advances Using Radial FLASH. Journal of Magnetic Resonance Imaging 2010; 31:101-9. [17] Duerk JL, Lewin JS, Wu DH. Application of keyhole imaging to interventional MRI: A simulation study to predict sequence requirements. Journal of Magnetic Resonance Imaging 1996; 6:918-24.

[18] Sequeiros RB, Klemola R, Ojala R, Jyrkinen L, Vaara T, Tervonen O. Percutaneous MR-guided discography in a low-field system using optical instrument tracking: A feasibility study. Journal of Magnetic Resonance Imaging 2003; 17:214-9. 


\section{Figure legends}

Fig. 1. Process of the simulation experiments. Starting with an original image, the original raw data was got by 2DFT. Then a simulated needle was "inserted". Selected views were updated and a new image was reconstructed. By replacing the original image with the updated image and advancing the needle at the same time, repetition of a cycle started. Besides, for every updated image, another simulated needle was attached for reference purpose.

Fig. 2. The sampling patterns of four updating schemes. Note that the exact numbers of lines in the images are not accurate. (a) Repeatedly single-division updating. The division size is $40 \%$. (b) AUSEK. Alternately the dotted lines or the solid lines get updated. The division size is $40 \%$. (c) Interleaved two-turn updating. Alternately the solid lines or the dotted lines get updated. $80 \%$ were updated in two turns. (d) Interleaved three-turn updating. In turns, the solid lines or the dotted lines or the dashed lines get updated. $80 \%$ were updated in three turns.

Fig. 3. The reconstructed images for four updating schemes. (a) Repeatedly singledivision updating. (b) AUSEK. (c) Interleaved two-turn updating scheme. (d) Interleaved three-turn updating scheme.

Fig. 4. The reconstructed images for four different levels of shifting. The sizes of all four are the same as $40 \%$. (a) $20 \% / 20 \%$. (b) $10 \%$ / 30\%. (c) $5 \% / 35 \%$. (d) $0 \% / 40 \%$.

Fig. 5. The reconstructed images for four different levels of division size. The smaller updating percentage in either half of k-space is fixed at $0 \%$. (a) $20 \%$. (b) $30 \%$. (c) $40 \%$. (d) $50 \%$.

Fig. 6. Images obtained during needle insertion into a phantom using AUSEK with FLASH. The division size was 40\% and the shifting level was 10\% / 30\%. The dark dot was assumed as the target. The tip position is marked in all the images. (a) Image of the fully-sampled reference scan. (b) Image updated immediately following one needle advance. (c) Image updated immediately following another needle advance. (d) Image of a fully-sampled scan when the needle hit the target. 
Fig. 7. Images obtained during needle insertion into a pig thigh using AUSEK with FLASH. The division size was $40 \%$ and the shifting level was $10 \%$ / 30\%. The small bone (kneecap) was chosen as the target. The tip position is marked in all the images. (a) Image of the fully-sampled reference scan. (b) Image updated immediately following one needle advance. (c) Image updated immediately following another needle advance. (d) Image of a fully-sampled scan when the needle hit the target. 


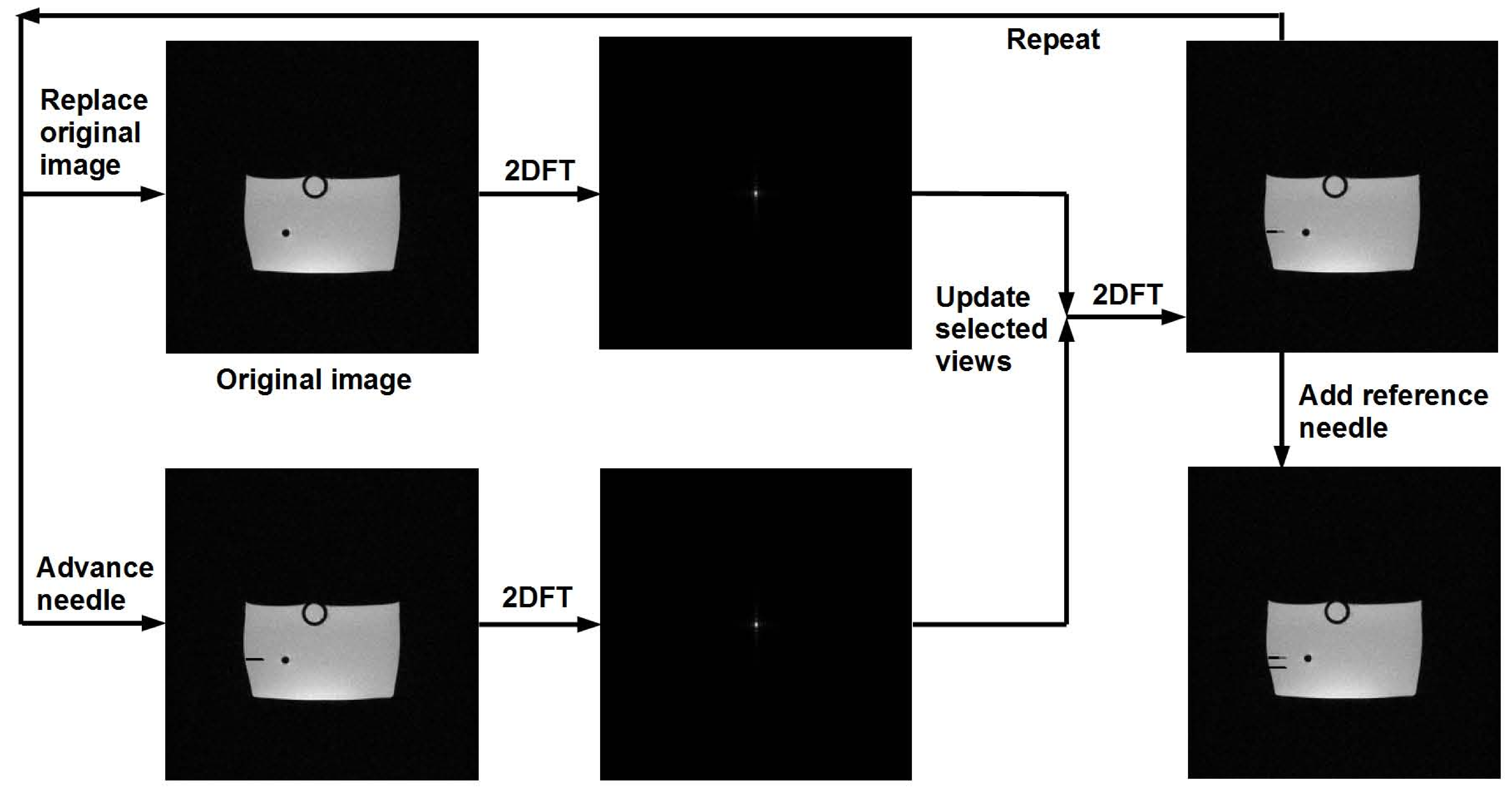



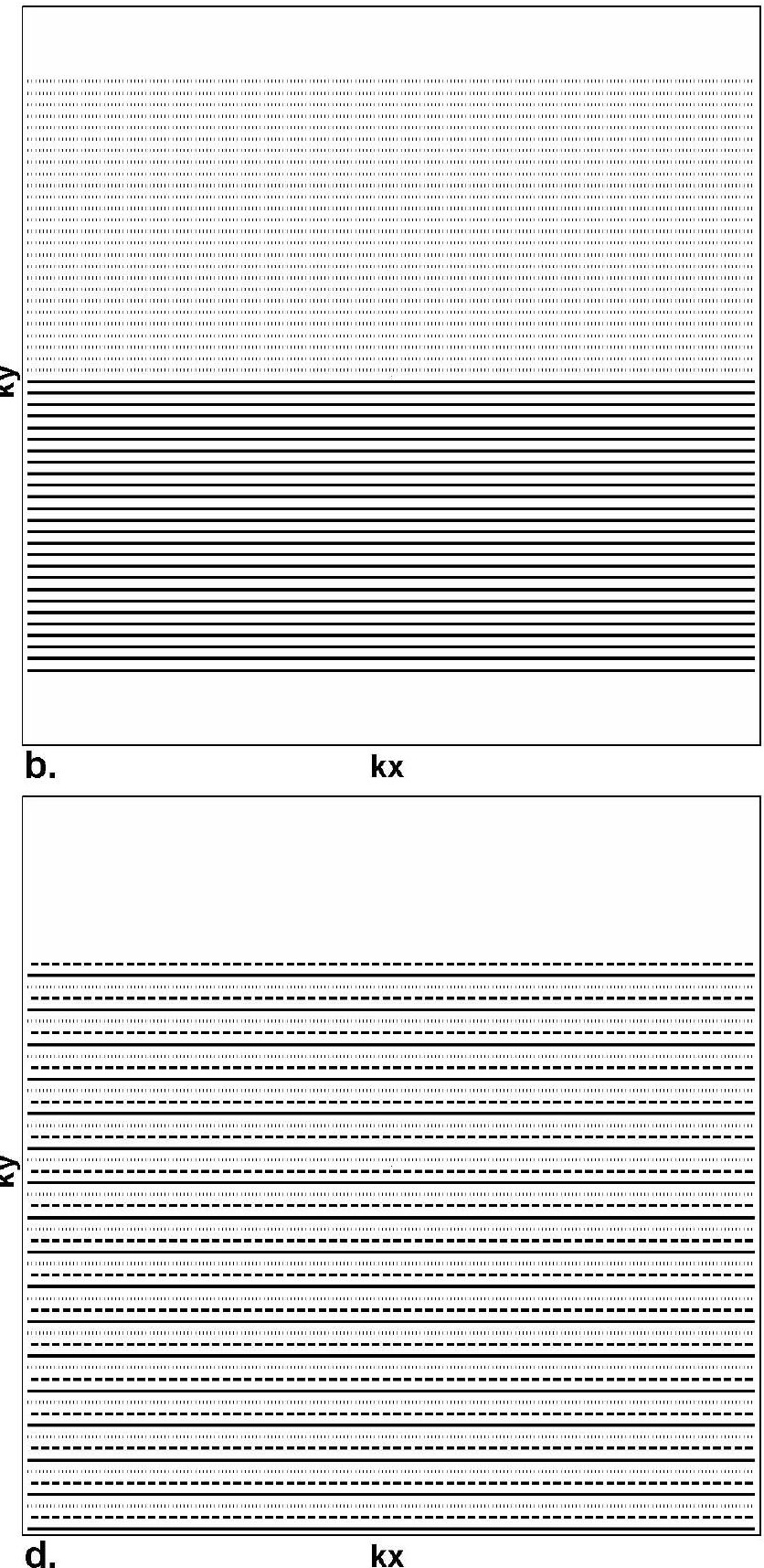

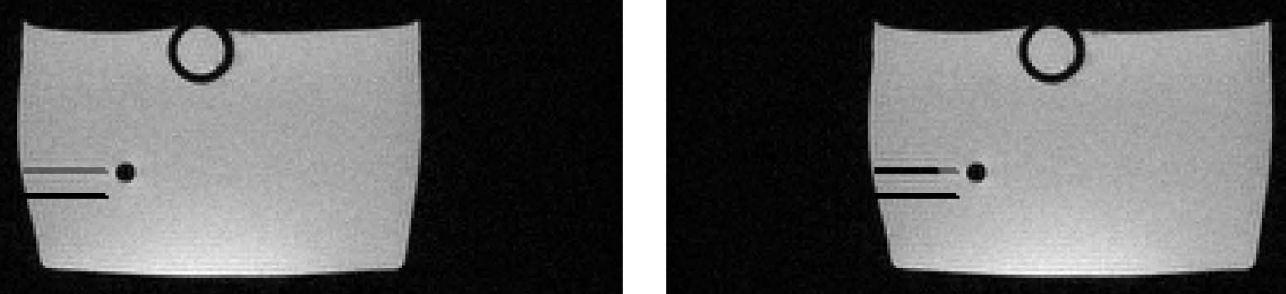

a.

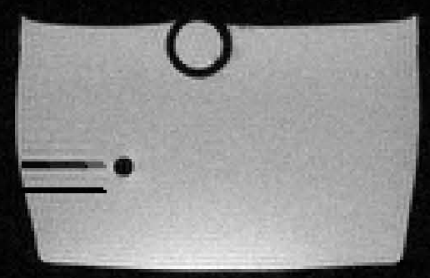

b.

\section{O}

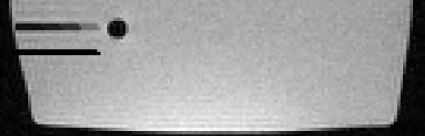



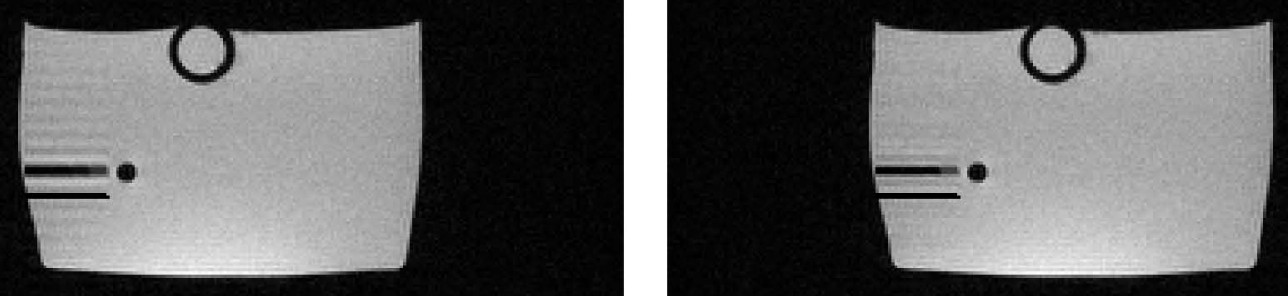

a.

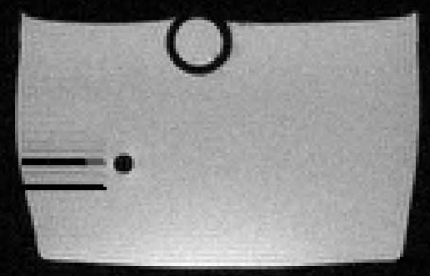

b.

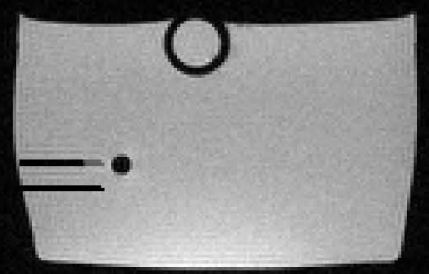

d. 

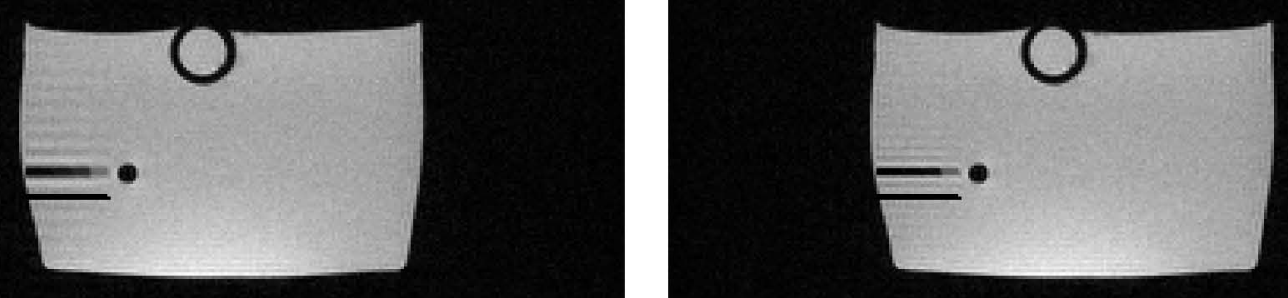

a.

b.
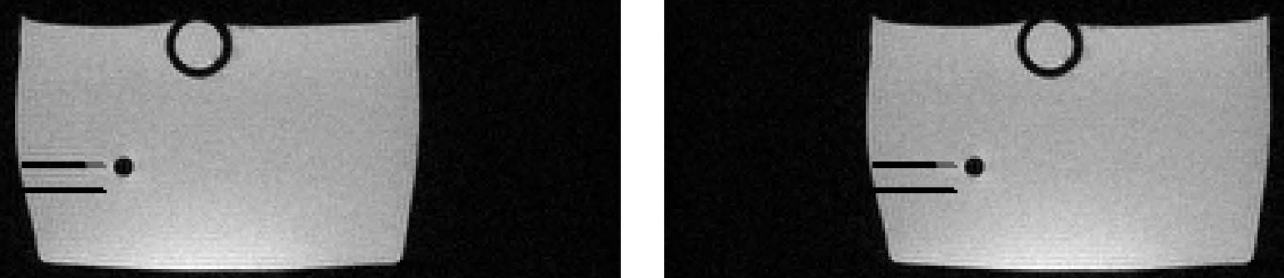

c. 

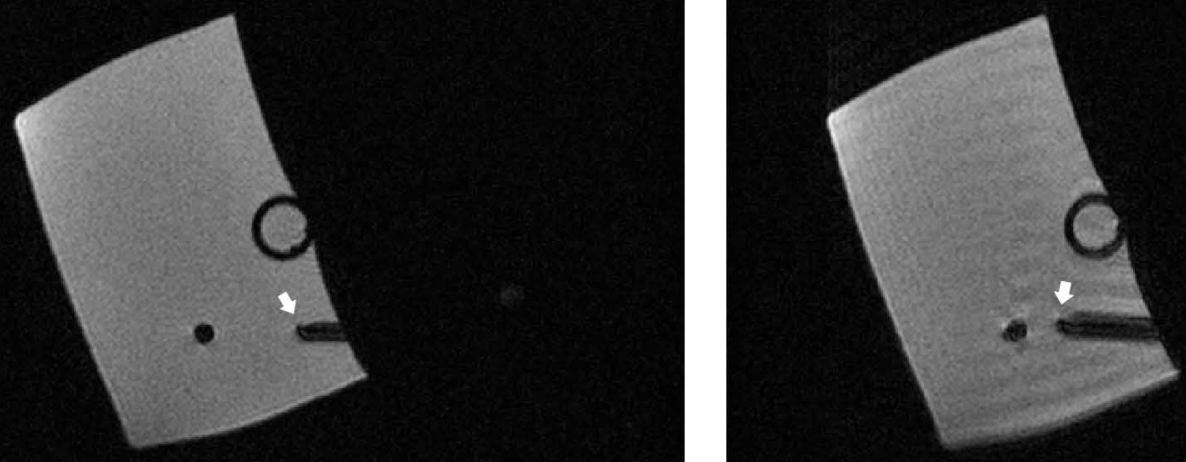

a.

b.
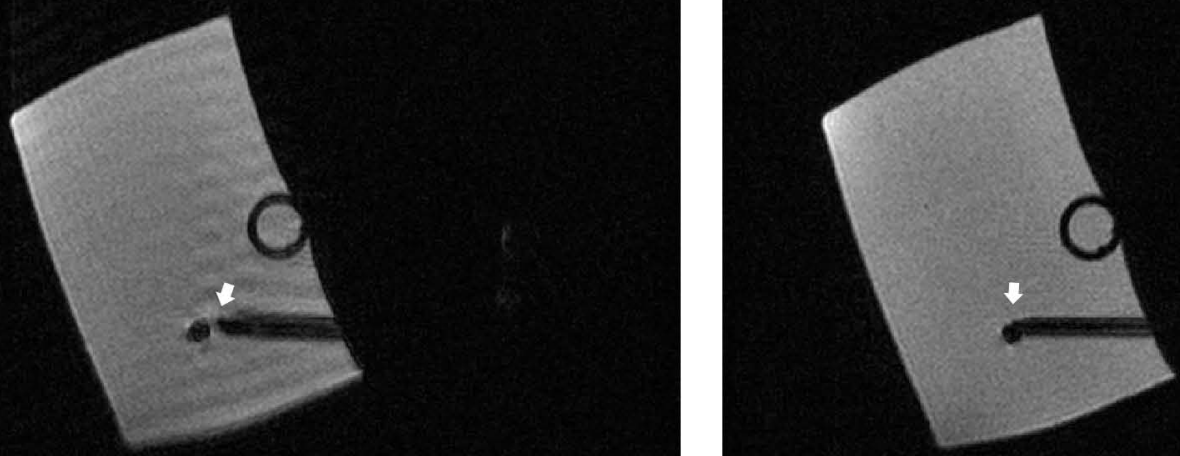

c.

d. 

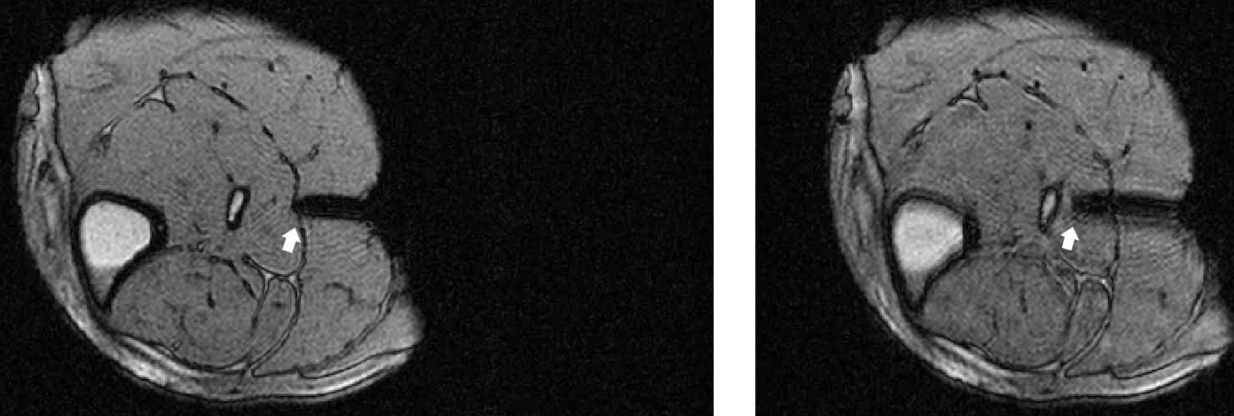

a.

b.
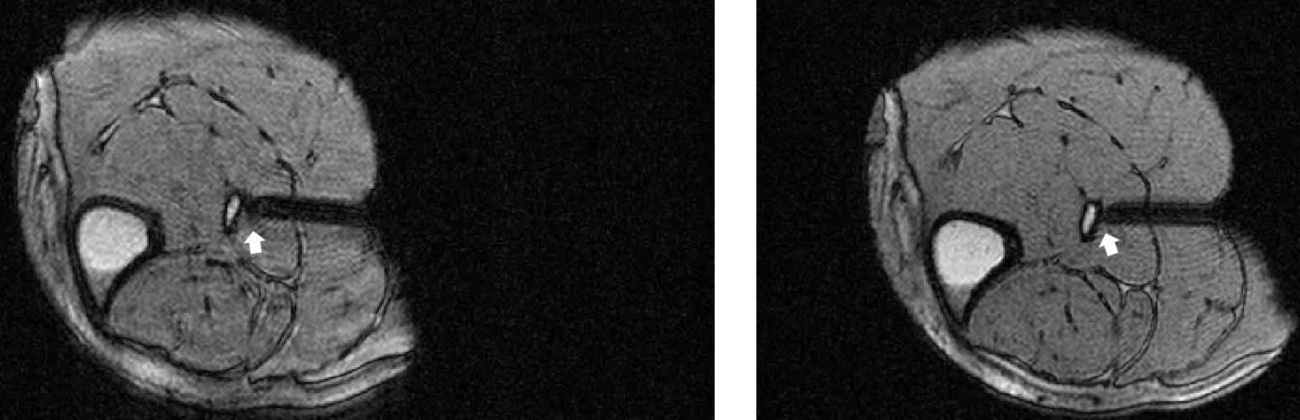

C.

d. 
Table 1. Quantitative evaluation of reconstruction errors of the experiments with four different updating schemes

\begin{tabular}{lllll}
\hline & $\begin{array}{l}\text { Single- } \\
\text { division }\end{array}$ & AUSEK & $\begin{array}{l}\text { Interleaved } \\
\text { Two-turn }\end{array}$ & $\begin{array}{l}\text { Interleaved } \\
\text { Three-turn }\end{array}$ \\
\hline Image including needle pixels & 0.0106 & 0.0053 & 0.0072 & 0.0081 \\
\hline Needle part including needle pixels & 0.1777 & 0.0884 & 0.0899 & 0.1180 \\
\hline Image excluding needle pixels & 0.0009 & 0.0012 & 0.0050 & 0.0042 \\
\hline Needle part excluding needle pixels & 0.0141 & 0.0171 & 0.0243 & 0.0208 \\
\hline $\begin{array}{l}\text { Note.- RMS differences of different parts between the reconstructed images and the original } \\
\text { one are shown. The details about every scheme can be found in the 2.3.1. }\end{array}$ \\
\hline
\end{tabular}


Table 2. Quantitative evaluation of reconstruction errors of the experiments with four different levels of shifting

\begin{tabular}{lllll}
\hline & $20 \% / 20 \%$ & $10 \% / 30 \%$ & $5 \% / 35 \%$ & $0 \% / 40 \%$ \\
\hline Image including needle pixels & 0.0052 & 0.0046 & 0.0048 & 0.0053 \\
\hline Needle part including needle pixels & 0.0840 & 0.0748 & 0.0790 & 0.0876 \\
\hline Image excluding needle pixels & 0.0042 & 0.0028 & 0.0021 & 0.0011 \\
\hline Needle part excluding needle pixels & 0.0748 & 0.0485 & 0.0334 & 0.0172
\end{tabular}

Note.-RMS differences of different parts between the reconstructed images and the original one are shown. The shifting level is denoted as the smaller updating percentage in either half of $\mathrm{k}$-space / the larger updating percentage in either half of $\mathrm{k}$-space. The division sizes for four levels are the same as $40 \%$. 
Table 3. Quantitative evaluation of reconstruction errors of the experiments with four different levels of division size

\begin{tabular}{llllc}
\hline & $20 \%$ & $30 \%$ & $40 \%$ & $50 \%$ \\
\hline Image including needle pixels & 0.0072 & 0.0057 & 0.0053 & 0.0052 \\
\hline Needle part including needle pixels & 0.1194 & 0.0941 & 0.0876 & 0.0874 \\
\hline Image excluding needle pixels & 0.0032 & 0.0021 & 0.0011 & 0.0006 \\
\hline Needle part excluding needle pixels & 0.0559 & 0.0346 & 0.0172 & 0.0094 \\
\hline $\begin{array}{l}\text { Note.- RMS differences of different parts between the reconstructed images and the original } \\
\text { one are shown. The level of division size is denoted by the percentage in either division. The } \\
\text { smaller updating percentage in either half of k-space is fixed at 0\%. }\end{array}$
\end{tabular}

